\title{
Maternal knowledge of optimal breastfeeding practices and associated factors in rural communities of Arba Minch Zuria
}

\author{
Dessalegn Tamiru, Shikur Mohammed \\ Public Health Department, Arbaminch University, Ethiopia \\ Email address: \\ dassutami@yahoo.com (D. Tamiru),m_shikur@yahoo.com (S. Mohammed)
}

\section{To cite this article:}

Dessalegn Tamiru, Shikur Mohammed. Maternal Knowledge of Optimal Breastfeeding Practices and Associated Factors in Rural Communities of Arba Minch Zuria, International Journal of Nutrition and Food Sciences. Vol. 2, No. 3, 2013, pp. 122-129.

doi: $10.11648 /$ j.ijnfs.20130203.15

\begin{abstract}
:
Introduction: Breastfeeding is one of the components of Primary Health Care and considered as natural practices in Ethiopia. However, a wide range of harmful infant feeding practices is documented even after implementations of infant and young child feeding guidelines in 2004. Therefore the major objective of this study was to assess maternal knowledge about optimal breastfeeding practices and associated factors in rural communities of Arba Minch Zuria.

Methods: A cross sectional community based study was carried out from January to February, 2012 in Arba Minch Zuria. Quantitative data were collected from 383 mothers supplemented with qualitative data from 10 key informants. Data were analyzed using SPSS version 16.0. Binary logistic regressions were used to see the strength of association between independent and dependent variables using odds ratios and $95 \%$ of confidence intervals. Finally a multivariate logistic regression analysis was made to identify the predictors of maternal knowledge about optimal breastfeeding practices.

Results: Breastfeeding was considered as a natural gift in Arba Minch Zuria. More than half of mothers (57.2\%) initiated breastfeeding within the first hour of delivery and $213(55.6 \%)$ were exclusively breastfed their children for 6 months. Three-hundred forty one (89\%) mothers gave colostrum though a small number of mothers considered colostrum as an expired breast milk and discarded it. Maternal knowledge about optimal breastfeeding was positively associated with paternal education level, total number of births, attending antenatal care, having the radio, using family planning and giving birth by health workers. This study also showed there is a positive relationship between maternal knowledge of optimal breastfeeding with exclusive breastfeeding and timely introduction of complementary food.

Conclusions: Findings from this study showed that maternal knowledge was directly related with paternal education level, attending antenatal care, having the radio, using family planning and giving birth by health workers. Maternal knowledge had a significant contribution in the promotion of optimal child feeding practices. Promotion of strong community based education and support to ensure optimal infant and young child feeding is recommended for the whole communities with health workers and community leaders to provide counseling and support related to infant and young child feeding practices.
\end{abstract}

Key words: Optimal, Knowledge, Factors, Arba Minch

\section{Introduction}

Globally, $60 \%$ of the infant and young child deaths occur due to inappropriate infant feeding practices and infectious disease where two-thirds of these deaths are attributable to sub-optimal breastfeeding practices $[1,2]$. Poor nutrition is not always only the result of lack of food, but it can be due to lack of knowledge about optimal feeding practices and provision of poor quality of food $[1,3]$. Clear-cut programs are needed especially in developing countries to provide a basic service and support for infants and young children to promote optimal breastfeeding and complementary feeding practices as they are essential to meet the nutritional needs of this vulnerable group $[1,4,5]$.

In many developing countries infants and young children are most vulnerable to malnutrition because of lack of knowledge how to feed a child and infectious diseases $[1,2$, $6,8]$. Consequently, large numbers of children are suffering 
from a wide-range of malnutrition manifested in stunted growth, wasting and micronutrient deficiencies [1, 2, 9].

Optimal newborn and infant feeding practices are major determinant of short and long-term health outcomes in individuals and social development. Children who are not breastfed properly have repeated infections, grow less and are more likely to die by the age of one month than children who receive at least some breast milk [3, 6]. Many observational studies showed that maternal knowledge of optimal child feeding practices like exclusive breastfeeding for six months, continued partially breastfeeding and the timely transition to adequate complementary food is basic to deliver physiological and economic benefits to mothers and to keep health of a child $[7,8]$.

Breastfeeding is nearly universal in Ethiopia. However, large numbers of mothers, both urban and rural, do not practice appropriate breastfeeding and complementary feeding behavior. In Ethiopia $57 \%$ of all under-five deaths is highly associated with abrupt cessation of breastfeeding and infectious diseases, but it is closely linked to gap of knowledge how to feed appropriately and food insecurity $[5,8,9]$. A recent report showed that $27 \%$ of mothers early provide water, butter and various types of food to feed their children, thereby reducing the percentage of exclusively breastfeed and increasing the percentage of receiving complementary food at very young age [9]. Nationally, $50.6 \%$ of mothers start breastfeeding within 1 hour of child birth and only about $38 \%$ mothers exclusively breastfed their children for 6 months [7, 8, 9, 10].

Generally, infant and young child feeding is a complex issue that has implications not only for an infant's nutritional and health status, but also affects infant's psychological development and the development of proper eating habits $[7,10]$. Although breastfeeding is one of the components of Primary Health Care in Ethiopia, a wide range of harmful infant feeding practices are documented even after implementations of infant and young child feeding recommendations. Therefore this study aimed to assess maternal knowledge about optimal breastfeeding practices and associated factors in rural communities of Arba Minch Zuria, Gamo Gofa Zone.

\section{Methods and Materials}

\subsection{Study Setting and Sample}

This cross sectional community based study was carried out from January to February, 2012 in Arba Minch Zuria. There are 31 kebeles (small community organization) in Arba Minch Zuria and 9 kebeles (small community organization) were randomly selected namely Chano Chalba, Kolla Shara, Shelle Mella, Kolla Shelle, Genta Meyche, Zigiti Merche, Gatse, Laka and Zeyesie Dembile. Arba Minch Zuria is one of the 112 districts in the Southern Nations, Nationalities and Peoples' Region of Ethiopia which is part of the Gamo Gofa Zone located in the Great East African Rift Valley [9, 19].
The study included mothers who had children under 2 years and permanent resident of selected kebeles. Motherchild index were identified using the most recent population registration data base. The simple random sampling technique was used to take the mothers-child pairs from each selected Kebele. The sample size was calculated using a formula for estimation of a single proportion as follows:

$$
\mathrm{n}=\frac{(\mathrm{Z} \alpha / 2)^{2} \mathrm{p}(1-\mathrm{p})}{\mathrm{d}^{2}}
$$

Where $\mathrm{Z}=$ Standard normal variable at $95 \%$ confidence level (1.96), $\mathrm{P}=$ Estimated proportion of optimal breastfeeding, $50 \%$, d $=0.05$ (5\% margin of error) [20]

10 key informants were selected purposely for in-depth interviews to capture their first-hand knowledge about a topic of interest. The selection was done based on their role in the community.

\subsection{Data Collection Procedure and Statistical Analysis}

Interviewer administered questionnaire and in-depth interview was used to collect questions concerning sociodemographics, maternal and child characteristics, child feeding practices and environmental health. Furthermore, women's knowledge of optimal child feeding practices and socio-cultural influences of child feeding were also included in the questionnaire. In-depth interview was used to generate descriptions of women's knowledge, experiences and perceptions of child feeding. Open-ended questions were used to follow research themes, then followed up using the participants' own words and phrases as a means of generating further information. Interviews were conducted at participant's home by audio tape recorder after getting consent from each study participants. Both quantitative and qualitative questionnaires were prepared in English and translated to Amharic by language experts and then translated back to English language by a third person to ensure the consistency. Finally data collectors were selected depending on their abilities of speaking the local language and training was given to them. The questionnaires were pre-tested. Based on a pretest result additional adjustment was made in terminologies, wording, and formatting of the questionnaire.

The data were checked for missing values and outliers, and analyzed using SPSS (SPSS Inc. version 16.0, Chicago, Illinois). Descriptive statistics were presented using standard statistical parameters such as percentages, means and standard deviations. Binary logistic regressions were used to see the strength of association between independent and dependent variable using odds ratios and 95\% of confidence intervals. All tests were two-sided. Finally a multivariate logistic regression analysis was made to identify the predictors of maternal knowledge about optimal child feeding practices. We report the results as adjusted odd ratios (AOR) and 95\% confidence intervals.

Qualitative data were analyzed after comparing collected data if the various people get the same impression and if 
there were differences and the reasons why. Then, it was analyzed separately depending on the answer of the respondents. The interviewed data were analyzed by system of open coding which involves sorting the data into analytical categories by breaking down, examining, comparing and categorizing data. Then categories of data were compared and contrasted to generate themes from the analysis and discussion. Finally, the result was presented in triangulation with the quantitative findings.

In this study maternal knowledge of optimal breastfeeding was defined as if the mother of a child had information about initiation of breastfeeding within one hour of delivery, giving colostrum, exclusive breastfeeding for 6 months, introduction of complementary food at 6 months, continued breastfeeding to 2 years and appropriate breastfeeding skills.

\subsection{Ethical Consideration}

The study was conducted after getting official permission from an ethical clearance committee of Arba Minch University, College of Medicine and Health Sciences. Then data were collected after getting official permission from Arba Minch Zuria Woreda Administration. Informed verbal consent was obtained from each study participant. Each respondent was informed about the objective of the study and privacy during the interview.

\section{Results}

Complete response was obtained from 383(99.74\%) of respondents. The majority of the study participants did not attend formal education (61.4\%). The mean ( \pm SD) age of the mothers was 29.08 years $( \pm 6.65)$ the range being from 15 to 46 years (Table 1 ).

Table 1: Socio-demographic characteristics of mothers in Arba Minch Zuria, 2012

\begin{tabular}{lll}
\hline Variable & Frequency (n) & Percent (\%) \\
\hline Marital status & \\
Married & 376 & 98.2 \\
Unmarried & 7 & 1.8 \\
Maternal education & & \\
No education & 235 & 61.4 \\
Reading and writing & 79 & 20.6 \\
Elementary and above & 69 & 19 \\
Mothers' occupation & & \\
Housewife & 381 & 99.5 \\
Others & 2 & 0.5 \\
Family size & & \\
$<5$ & 121 & 31.6 \\
$\geq 5$ & 262 & 68.4 \\
Maternal age (in years) & & \\
15-20 & 28 & 7.3 \\
$21-25$ & 96 & 25.1 \\
$26-30$ & 134 & 35 \\
$>31$ & 125 & 32.6 \\
Attended ANC & & 83.3 \\
Yes & 319 & 16.7 \\
No & 64 & \\
\hline
\end{tabular}

\begin{tabular}{lll}
\hline Family planning & & \\
Utilization & & \\
Yes & 237 & 61.9 \\
No & 146 & 38.1 \\
Delivery assistant & & \\
TBA & 197 & 51.4 \\
Health workers & 41 & 10.7 \\
Others* & 145 & 37.9 \\
Delivery type & & \\
Caesarean section & 6 & 1.6 \\
Vaginal delivery & 377 & 98.4 \\
Place of delivery & & \\
Home & 370 & 96.6 \\
Health center and & 13 & 3.4 \\
Hospital & & \\
Total number of birth & 146 & 38.1 \\
2 & 237 & 61.9 \\
F2 & & \\
Farm land owners & 94 & 75.5 \\
No & 289 & 24.5 \\
Yes & & 61.1 \\
Religion & 234 & 36.0 \\
Protestant & 138 & 2.9 \\
Orthodox & 11 & \\
Others** & & \\
\hline
\end{tabular}

*Relatives, Neighbors, Family; ** Muslim, Traditional

Breastfeeding is considered as a natural gift in the rural communities of Arba Minch Zuria. All mothers believed that an infant should get breast milk since it is a natural gift for them. Some verbatim expressions of the mothers which represent this idea where:

"...breast milk is natural gift; it is easily available and the digestible type of food for newborn infants"

The majority of mothers $(57.2 \%)$ started breastfeeding within the first hour of delivery though others delayed due to lack of time and some traditional beliefs. A large proportion of mothers (89 \%) considered provision of colostrum as vaccines given to infants to prevent diseases. Similarly in-depth interview with mothers showed a majority of mothers reported that they have given first milk for their child and have good attitude toward provision of colostrum for newborn infants.

Twenty 28 years old mothers said;

"Colostrum is an important diet for infants as it contains all important nutrients and used to prevent against disease..."

However, a small number of mothers discarded colostrum since they had no basic information about dietary importance of colostrum (Table 2). An in-depth interviews with 26 years old mothers showed that colostrum is expired breast milk and it is not good for child health.

"...colostrum an expired yellowish substance which can cause disease to newborn infants...”

Two-hundred thirteen mothers (55.6\%) exclusively breastfed their children for 6 months. Some mothers $(41.18 \%)$ provided water to cleanse the stomach of an infant. Instead of breast milk $8(23.53 \%)$ of mothers provided cow's milk soon after delivery (Table 2). However the findings from in-depth interviews showed that currently there is no provision of any forms of water. 
Thirty years traditional birth attendant said;

"Previous time water will be given to cleansing and treat colic disease, but currently there is no such practice."

Table 2: Breastfeeding patterns in Arba Minch Zuria, 2012

\begin{tabular}{|c|c|c|}
\hline Variables & Frequency (n) & Percent (\%) \\
\hline \multicolumn{3}{|c|}{ Breastfeeding initiation } \\
\hline Within one hour & 219 & 57.2 \\
\hline After one hour & 164 & 42.8 \\
\hline \multicolumn{3}{|l|}{ Giving colostrum } \\
\hline Yes & 341 & 89 \\
\hline No & 42 & 11 \\
\hline \multicolumn{3}{|c|}{ Breastfeeding Frequency } \\
\hline$<8$ & 214 & 55.9 \\
\hline$\geq 8$ & 169 & 44.1 \\
\hline \multicolumn{3}{|c|}{ Duration of EBF in month } \\
\hline $0-1$ & 32 & 8.4 \\
\hline $2-3$ & 28 & 7.3 \\
\hline $4-5$ & 60 & 15.7 \\
\hline $0-6$ & 213 & 55.6 \\
\hline$\geq 7$ & 50 & 13.1 \\
\hline \multicolumn{3}{|c|}{ Giving additional food } \\
\hline $0-2$ & 40 & 10.4 \\
\hline $3-4$ & 81 & 21.1 \\
\hline $5-6$ & 195 & 50.9 \\
\hline$\geq 7$ & 67 & 17.5 \\
\hline \multicolumn{3}{|c|}{ Type of pre-lacteal food } \\
\hline Milk & 8 & 23.53 \\
\hline Water & 14 & 41.18 \\
\hline Others & 12 & 35.29 \\
\hline \multicolumn{3}{|l|}{ Utensil of feeding } \\
\hline Bottle & 8 & 2.1 \\
\hline Cup & 136 & 35.5 \\
\hline Spoon & 63 & 16.4 \\
\hline No utensil & 176 & 46 \\
\hline
\end{tabular}

Multivariable regression model showed that maternal knowledge of optimal breastfeeding was positively associated with paternal education level, total number of births, attending antenatal care, having the radio, using family planning and giving birth by health workers (Table $3)$.

This study showed that paternal education level is significantly associated with maternal knowledge of optimal breastfeeding practices. Women whose husbands did not attend any formal education had less knowledge about optimal breastfeeding practices $(\mathrm{AOR}=2.92[1.28$, 6.63]) compared to whose husbands attended primary school and above. Qualitative data also showed that mothers who got information about breastfeeding were appropriately feed their children.

One Traditional Birth Attendant said:

"... we have got the basic concepts about impacts of traditional malpractices and sub-optimal breastfeeding from health workers and our family."

Total number of birth was also significantly associated with maternal knowledge of optimal breastfeeding practices. Mothers who born two and less than two children had less lack of knowledge about optimal child feeding practices $(\mathrm{AOR}=0.47[0.29,0.76])$ compared to who born two and more than children.

Having radio had a significant role in the promotion of maternal knowledge of optimal breastfeeding practices. Mothers (families) who had radio were less lack of knowledge about optimal breastfeeding practices $(\mathrm{AOR}=$ $0.56[0.33,0.95])$. In-depth interview with mothers also showed that health education had a significant role in the promotion of optimal breastfeeding practices.

Family planning utilization was positively associated with maternal knowledge of optimal breastfeeding practices. Mothers who were not using family planning had less knowledge of optimal breastfeeding $(\mathrm{AOR}=0.47[0.27$, $0.83]$ ) compared to who were using.

Table 3: Factors Associated with Maternal Knowledge of Optimal Breastfeeding in Arba Minch Zuria, 2012

\begin{tabular}{|c|c|c|c|c|}
\hline \multirow{2}{*}{ Variable } & \multicolumn{4}{|c|}{ Mothers' had no knowledge of optimal breastfeeding } \\
\hline & $\mathrm{n}$ & $\mathrm{OR}(95 \% \mathrm{CI})$ & $\mathrm{AOR}(95 \% \mathrm{CI})$ & P-Value \\
\hline \multicolumn{5}{|l|}{ Family size } \\
\hline 5 & 57 & $0.79(0.51,1.22)$ & $1.34(0.73,2.37)$ & 0.361 \\
\hline$>5$ & 59 & 1 & 1 & \\
\hline \multicolumn{5}{|l|}{ Marital status } \\
\hline Married & 116 & $2.68(0.32,22.49)$ & $2.68(0.28,25.77)$ & 0.395 \\
\hline Unmarried & 1 & 1 & 1 & \\
\hline \multicolumn{5}{|l|}{ Mothers' Educational } \\
\hline No education & 77 & $1.38(1.76,2.52)$ & $0.48(0.19,1.21)$ & 0.117 \\
\hline Reading and Writing & 22 & $1.09(0.52,2.27)$ & $0.54(0.19,1.48)$ & 0.229 \\
\hline Elementary and abo & 18 & 1 & 1 & \\
\hline \multicolumn{5}{|l|}{ Father's education } \\
\hline No education & 65 & $2.22(1.30,3.79)^{* *}$ & $2.92(1.28,6.63)$ & 0.011 \\
\hline Reading and Writing & 27 & $2.20(1.16,4.19)^{*}$ & $3.08(0.26,7.50)$ & 0.063 \\
\hline Elementary and above & 25 & 1 & 1 & \\
\hline \multicolumn{5}{|l|}{ Total birth } \\
\hline 2 & 31 & $0.47(0.29,0.76)^{* *}$ & $0.39(0.20,0.75)$ & 0.005 \\
\hline$>2$ & 86 & 1 & 1 & \\
\hline \multicolumn{5}{|l|}{ Under five children } \\
\hline 1 & 73 & $0.60(0.38,0.96)^{*}$ & $0.75(0.44,1.29)$ & 0.301 \\
\hline
\end{tabular}




\begin{tabular}{|c|c|c|c|c|}
\hline Religion & & & & \\
\hline Protestant & 69 & $0.35(0.1,1.18)$ & $0.44(0.11,1.75)$ & \\
\hline Orthodox & 42 & $0.37(0.11,1.26)$ & $0.43(0.11,1.75)$ & 0.245 \\
\hline Others & 6 & 1 & 1 & 0.241 \\
\hline \multicolumn{5}{|l|}{ Maternal age } \\
\hline $15-20$ & 8 & $0.97(0.2,3.24)$ & $2.37(0.57,9.86)$ & 0.236 \\
\hline $21-25$ & 63 & $0.92(0.36,2.31)$ & $1.66(0.56,4.96)$ & 0.360 \\
\hline $26-30$ & 39 & $1.53(0.58,4.02)$ & $1.71(0.58,5.08)$ & 0.334 \\
\hline$>31$ & 7 & 1 & 1 & \\
\hline \multicolumn{5}{|l|}{ Ethnicity } \\
\hline Gamo & 89 & $0.57(0.33,0.99)$ & $0.49(0.25,0.99)$ & 0.046 \\
\hline Wolaita & 9 & $1.35(0.48,3.78)$ & $2.73(0.79,9.44)$ & 0.113 \\
\hline Others & 26 & 1 & 1 & \\
\hline \multicolumn{5}{|l|}{ ANC follow up } \\
\hline Yes & 85 & $0.36(0.21,0.63)^{* * *}$ & $0.45(0.24,0.86)$ & 0.015 \\
\hline No & 32 & 1 & 1 & \\
\hline \multicolumn{5}{|l|}{ Child sex } \\
\hline Female & 51 & $0.82(0.53,1.27)$ & $0.80(0.48,1.32)$ & 0.378 \\
\hline Male & 66 & 1 & 1 & \\
\hline \multicolumn{5}{|l|}{ Birth place } \\
\hline Home & 116 & $5.48(0.70,42.65)$ & $10.68(0.89,12.18)$ & \\
\hline Health post & 1 & 1 & 1 & 0.062 \\
\hline \multicolumn{5}{|l|}{ Delivery assistant } \\
\hline TBA & 67 & $1.50(0.94,2.42)$ & $1.61(0.92,2.80)$ & 0.093 \\
\hline HEW & 13 & $1.36(0.636,2.89)$ & $3.56(1.31,9.63)$ & 0.013 \\
\hline Relatives \& Neighbor & 37 & 1 & 1 & \\
\hline \multicolumn{5}{|l|}{ Having farm land } \\
\hline Yes & 90 & $1.12(0.67,1.87)$ & $1.03(0.56,1.90)$ & 0.917 \\
\hline No & 27 & 1 & 1 & \\
\hline \multicolumn{5}{|l|}{ Having radio } \\
\hline Yes & 42 & $0.49(0.31,0.77)^{* *}$ & $0.56(0.33,0.95)$ & 0.032 \\
\hline No & 75 & 1 & 1 & \\
\hline \multicolumn{5}{|l|}{ Used family Planning } \\
\hline Yes & 50 & $0.32(0.20,0.50)^{* * *}$ & $0.47(0.27,0.83)$ & 0008 \\
\hline No & 67 & 1 & 1 & 0.000 \\
\hline
\end{tabular}

Findings from this study showed that there is a relationship between maternal knowledge of optimal breastfeeding with appropriate breastfeeding practices. Mothers who had no knowledge of optimal breast feeding were $90 \%$ less exclusive breastfeeding $(\mathrm{AOR}=0.10(0.03$, $0.371)$ ) and $94 \%$ less ever breasted $(\mathrm{AOR}=0.06(0.01,0.65)$ compared to their counterparts. Mothers who had information about optimal breastfeeding were also more exclusive breastfeeding to one month $(\mathrm{AOR}=3.16(1.12$, 8.91) compared to who had no information. Similarly mothers who had knowledge of optimal breastfeeding were $46 \%$ more timely introduce complementary food $(\mathrm{AOR}=1.46(1.23,2.90)$ to who had no knowledge to six months (Table 4).

Table 4: Association of feeding practices and maternal knowledge of optimal breastfeeding in Arba Minch Zuria, 2012

\begin{tabular}{|c|c|c|c|c|}
\hline \multirow{2}{*}{ Variable } & \multicolumn{4}{|c|}{ Mothers' had knowledge of optimal breastfeeding } \\
\hline & $\mathbf{n}$ & $\operatorname{COR}(95 \% \mathrm{CI})$ & $\operatorname{AOR}(95 \% C I)$ & P-Value \\
\hline $\begin{array}{c}\mathrm{BF}^{1} \text { initiation } \\
\text { After one hour } \\
\text { Within one hour }\end{array}$ & $\begin{array}{l}57 \\
60\end{array}$ & $\begin{array}{c}0.71(0.46,1.10) \\
1\end{array}$ & $\begin{array}{c}0.80(0.49,1.33) \\
1\end{array}$ & 0.394 \\
\hline $\begin{array}{c}\text { Discarded colostrum } \\
\text { No } \\
\text { Yes }\end{array}$ & $\begin{array}{c}102 \\
15\end{array}$ & $\begin{array}{c}1.30(0.67,2.55) \\
1\end{array}$ & $\begin{array}{c}0.98(0.4,2.15) \\
1\end{array}$ & 0.961 \\
\hline $\begin{array}{c}\mathrm{EBF}^{2} \text { to } 6 \text { month } \\
\text { No } \\
\text { Yes }\end{array}$ & $\begin{array}{l}97 \\
20\end{array}$ & $\begin{array}{c}0.07(0.03,0.22) \\
1\end{array}$ & $\begin{array}{c}0.10(0.03,0.37) \\
1\end{array}$ & 0.001 \\
\hline $\begin{array}{c}\text { Ever breastfed } \\
\text { No } \\
\text { Yes }\end{array}$ & $\begin{array}{c}6 \\
111\end{array}$ & $\begin{array}{c}0.07(0.01,0.59) \\
1\end{array}$ & $\begin{array}{c}0.06(0.01,0.65) \\
1\end{array}$ & 0.020 \\
\hline $\begin{array}{c}\mathrm{BF}^{1} \text { at night } \\
\text { No } \\
\text { Yes }\end{array}$ & $\begin{array}{c}4 \\
113\end{array}$ & $\begin{array}{c}0.76(0.22,2.66) \\
1\end{array}$ & $\begin{array}{c}3.05(0.51,18.16) \\
1\end{array}$ & 0.220 \\
\hline $\begin{array}{c}\mathrm{EBF}^{2} \text { in month } \\
0-1 \\
2-3\end{array}$ & $\begin{array}{c}19 \\
2\end{array}$ & $\begin{array}{l}2.84(1.14,7.10) \\
0.97(0.16,5.85)\end{array}$ & $\begin{array}{l}3.16(1.12,8.91) \\
0.27(0.02,3.00)\end{array}$ & $\begin{array}{l}0.030 \\
0.286\end{array}$ \\
\hline
\end{tabular}




\begin{tabular}{|c|c|c|c|c|}
\hline $4-5$ & 29 & $2.01(0.92,4.40)$ & $1.94(0.76,4.99)$ & 0.168 \\
\hline $0-6$ & 50 & $0.52(0.27,1.00)$ & $0.78(0.37,1.64)$ & 0.509 \\
\hline$\geq 7$ & 17 & 1 & 1 & \\
\hline \multicolumn{5}{|l|}{ Age of giving food } \\
\hline $0-2$ & 14 & $0.91(0.40,2.05)$ & $0.56(0.21,1.48)$ & 0.240 \\
\hline $3-4$ & 34 & $1.22(0.62,2.36)$ & $0.61(0.27,1.36)$ & 0.224 \\
\hline $5-6$ & 44 & $0.49(0.27,0.89)$ & $1.46(1.23,2.90)$ & 0.023 \\
\hline$\geq 7$ & 25 & 1 & 1 & \\
\hline \multicolumn{5}{|l|}{ Utensil of feeding } \\
\hline Bottle & 1 & $0.28(0.03,2.36)$ & $0.47(0.05,4.12)$ & 0.493 \\
\hline Cup & 49 & $1.12(0.70,1.79)$ & $1.19(0.69,2.03)$ & 0.533 \\
\hline Spoon & 8 & $0.29(0.13,0.65)$ & $0.34(0.14,1.83)$ & 0.119 \\
\hline Not instruments & 59 & 1 & 1 & \\
\hline
\end{tabular}

$\mathrm{EBF}=$ Exclusive Breastfeeding, $\mathrm{BF}=$ Breastfeeding

\section{Discussion}

Studies showed that maternal knowledge about optimal breastfeeding had a significant role in the promotion of optimal breastfeeding practices. In Ethiopia 57\% of infant and young child's death is significantly associated with sub-optimal breastfeeding practices. This might be due to lack of knowledge about optimal breastfeeding practices [5, $9,10]$. The fact that a large proportion of mothers practiced sub-optimal feeding practices after eight years of development of the national infant and young child feeding guideline indicates the need for strengthening the behavior change communication on optimal infant and young child feeding practices. This might be also an obstacle in the achievement of the fourth millennium development goal which intends to reduce infant and young child mortality by two-thirds from 1990 to 2015 [1, 9, 12].

Breastfeeding is considered as a natural gift in Arba Minch Zuria Woreda. An in-depth interview showed some mothers perceived breastfeeding as a natural gift though they could not feed appropriately due to field and home activities. Studies from different countries also showed that marketing and agricultural activities are found to be a constraint for optimal infant and young child feeding practices [11]. Despite national and international recommendations to promote exclusive breastfeeding practices only $55.5 \%$ of mothers were exclusively breastfeeding to 6 months in Arba Minch Zuria woreda which is relatively high compared with previously reported data of 2011 Ethiopian Demographic and Health Survey were $52 \%$ of mothers exclusively breastfed for 6 months [9]. About $42.7 \%$ of mothers initiated breastfeeding after one hour of delivery. This figure is low compared to findings of 2011 Ethiopian Demographic and Health Survey (48\%) and a study done in Bangladesh where $76 \%$ of mothers initiated breastfeeding after an hour of delivery[9, 13]. Similarly in-depth interview findings showed some mothers did not know when breastfeeding should be started and set socio-cultural factors as constraints to initiate breastfeeding.

More than half $(50.9 \%)$ of mothers introduced complementary food like cow's milk, fruits and vegetables after 5 months; because they thought that breast milk was insufficient. In-depth interviews with mothers also showed a majority of mothers introduced additional food as they believed that breast milk was insufficient to 6 months which is consistent with a study done in South Africa and Gambia were mothers provided additional food to satisfy babies or to put them to sleep $[12,14]$.

Some mothers (11\%) discarded colostrum as they considered it as water and cause of disease. This figure is low compared to national figures (23\%) [9]. Similar studies in developing countries indicated that some mothers discarded colostrum as they considered as water and causes of diseases [12, 15]. However, in-depth interview with mothers indicated majority of mothers considered colostrum preventive medicine.

The paternal educational level is positively associated with maternal knowledge of optimal breastfeeding practices. Women whose husbands did not attend any formal education had less knowledge about optimal breastfeeding practices compared to whose husband attended formal education. This might be due to paternal influences to feed a child as he learnt along with different courses. Studies also showed that parents and health workers have significant contribution in the promotion of optimal child feeding practices $[3,8,15]$. Studies from Kenya and Gambia similarly showed maternal education has significant contribution to alleviate sub-optimal child feeding practices $[7,12,16]$.

Mothers who gave birth less than or equal to two children were more appropriately fed their children compared to who gave many births. This might be due to an exposure to health education given by health workers at different occasion on child feeding practices and family planning utilization.

Having radio had a significant contribution in the promotion of maternal knowledge of optimal breastfeeding practices. Mothers (families) who had no radio where less knowledge about optimal breastfeeding practices. This might be due to education and advertisement given by media about child feeding practices. Studies in different countries also showed breastfeeding encouragement and advertisement through media had a significant role in the promotion of optimal breastfeeding practices $[2,12,15,16]$.

The findings of this study indicate the utilization of family planning is one of the contributing factors for maternal knowledge of optimal breastfeeding practices. 
Mothers who used family planning were more optimal child feeding than who did not use. This might be due to basic education which is given along with family planning by health extension workers to promote optimal child feeding practices. Findings from different countries also showed maternal exposure to the health education and advertisement about breastfeeding practices is determining factor for an optimal child feeding practices $[2,8,12,15$, $16]$.

Optimal breastfeeding knowledge was more common among mothers who were exclusively breastfed for 6 months. This indicates that basic education in the promotion of optimal breastfeeding should be encouraged. Findings from in-depth interviews similarly indicated mothers' knowledge of optimal breastfeeding is due to an exposure to health education given by health extension workers. This study indicated health education which is given at different occasion concerning about hygiene, complementary food and breastfeeding practices is one of the predetermining factors to promote optimal breastfeeding practices. In addition maternal knowledge of optimal child feeding has significant impact in timely introduction of complementary food. However, some mothers provided milk, water and some traditional drugs before they start breastfeeding since they believed that it prevents a child from stomachache. The study done in Tanzania, Bangladesh and Nigeria also indicated mothers gave additional food certain prelacteal feeds due to peer influence and some traditional beliefs as they considered it as a preventive measure against disease $[13,17,18]$.

Findings from this study have significant input in the promotion of optimal child feeding practices and the achievement of the millennium development in the reduction of child mortality. However, it shares the drawbacks of cross-sectional studies that are difficult to establish cause-effect relationship between dependent and independent variables. There might be also recall bias on child feeding practices as mothers could forget when they initiated breastfeeding and gave an additional food, but this bias was minimized by relating to events and training data collectors how to probe the mothers to remember what she fed.

\section{Conclusions}

Findings from this study showed that maternal knowledge was directly related with paternal education level, attending antenatal care, having the radio, using family planning and giving birth by health workers. Maternal knowledge had a significant contribution in the promotion of optimal child feeding practices. Promotion of strong community based education and support to ensure optimal infant and young child feeding is recommended for the whole communities with health workers and community leaders to provide counseling and support related to infant and young child feeding practices.
The following recommendations are suggested based on the results of the study.

$>$ Educating mothers about optimal child feeding practices at different occasion like a meeting, coffee ceremony and edirs is the good opportunity to enhance mothers' knowledge of child feeding practices.

$>$ Coordinate exclusive breastfeeding support and promotion with other programs in the health department, private and public health care systems and community organizations.

$>$ Behavioral change communications on traditional gatherings such as coffee ceremony, market days and the like should be strengthened in collaboration government and non-government organizations.

\section{Acknowledgements}

The authors would like to express their sincere gratitude to the Arba Minch University and study participants for their diligence and dedication in collecting and inputting a high quality data used for the study.

\section{References}

[1] UNICEF and World Health Organization. 2003. Global Strategy for Infant and Young Child Feeding, Geneva, Switzerland

[2] Bernadette et al. 2003. Special Issue Base World Health Organization Expert Consultation on Complementary Feeding. Food and Nutrition Bulletin; 24(1): 3-141.

[3] WHO.2003. Implementing the global strategy for infant and young child feeding. Department of Nutrition for Health and Development. Geneva

[4] Altrena G. et al. 2006. Infant and Young Child Feeding Update, ORC Macro Calverton, Maryland, USA.

[5] Central Statistical Authority [Ethiopia] and ORC Macro. 2005. Ethiopia Demographic and Health Survey 2005. Addis Ababa, Ethiopia and Calverton, Maryland

[6] Gretel H. et al. 2003. Improving feeding practices: Current patterns, common constraints, and the design of interventions. Division of Nutritional Sciences; 24(1): 45-57

[7] WHO. 2001. Report of the global consultation on Summary of guiding principles for complementary feeding of the breastfed child, Geneva Switzerland.

[8] Federal ministry of health. 2005. National strategy for child survival in Ethiopia, Addis Ababa Ethiopia, Family health department publications.

[9] Central Statistical Authority and ORC Macro. 2012. Ethiopia Demographic and Health Survey 2011. Addis Ababa, Ethiopia and Calverton, Maryland

[10] Federal Ministry of Health. 2004. National strategy for infant and young child feeding. Addis Ababa, Ethiopia.

[11] United Nations. 2008. The Millennium Development Goals. United Nations Department of Economic and Social Affairs (DESA). New York. 
[12] Erik Bohler et al. 2001. Promoting breastfeeding in rural Gambia: combining traditional and modern knowledge. Health policy and planning; 16(2):199-205.

[13] Haider et al. 2010. Breastfeeding in infancy: identifying the program-relevant issues in Bangladesh. International Breastfeeding Journal 5:21.

[14] R Kruger and GJ Gericke. 2002. A qualitative exploration of rural feeding and weaning practices, knowledge and attitudes on nutrition in South Africa. Public Health Nutrition; 6(2):217-223

[15] Tefera Belachew.2003. Human Nutrition for Health Science Students Jimma. Public Health Department.

[16] Kimani-Murage et al. 2011. Patterns and determinants of breastfeeding and complementary feeding practices in urban informal settlements, Nairobi Kenya. BMC Public Health, $11: 396$.
[17] Shirima, M Gebre-Medhin and T Greiner. 2001. Information and socioeconomic factors associated with early breastfeeding practices in rural and urban Morogoro, Tanzania. Acta Paediatr 90:936-942.

[18] Ijoritimi Oluwole Steve. 2006. Nutritional Status and feeding practices of infants low income Nursing Mothers in Ondo state, Nigeria. International Journal of Tropical Medicine; 1 (3): $123-129$

[19] USAID FEWS NET ACTIVITY. 2006. Southern Nation, Nationalities and People's Region, Ethiopia Livelihood Profiles

[20] Chapt T Le. 2003. Introductory Biostatistics. Comprehensive Cancer Center, University of Minnesota, United States of America 